\title{
Sachregister zu Band 83
}

Die fett gedruckten Zahlen bedeuten Eigenarbeiten. Bb. = Buchbesprechungen.

A

Ablatio retinae 139, 142, 143, 231, 232

Entstehung der $\cdot-130$

und Trauma 222

Hypotonie bei -217

diathermische Stichelung bei - 98, 189, 234

chirurgische Behandlung der - 123, 131, 138, 238

Acetylcholin bei Migraine oph-

thalmique 118 Airol, Massage der Bindehaut mit

- bei Trachom 115 Akkommodation 202 (Bericht) Akkommodationskrampf als

traumatische Neurose 137

- $\quad$ Bedeutung des - für die Ent-wicklung der Myopie 200.

Akrokephalo-Syndaktylie 48 Aktinomyces 302 Alkoholinjektionen in das Lid

bei Entropium 114 Allergie und Immunität 306 Allergielehre 246 Alterserscheinungen am Auge

47 Anaërobier am Auge 295 Anaphylaxie und Immunität 246,

307 Angioidstreifen am Augen-

hintergrunde 72 Angiomatosis retinae, beider-

seitige 244 Aniridie, Stammbaum von - 37 Anisometropie, myopische Fun-

dusdegeneration bei - 252 Arachnodaktylie zugleich mit

Ektopia lentis 37 Argyrosis conjunctivae, erb-

liche 36 Arsenvergiftung 315 Arteria communicans anterior, Aneurysmen der - 243 Arthus-

Phänomen 307 Aspergillus fumigatus 302 Astigmatismus 55

- $\quad$ und Myopie 200, 201Atophanyl bei Ophthalmia sym-

pathica 117 Atropin, retrobulbäre Einsprit-zungen von - bei Sehnerven-atrophie 117, 134

Augenbrauen, Form und Farbe der -36

Augenf art $>$ e, Vererbung der -38

Augenhintergrund, myopische Degeneration des - bei Anisometropie 252

Augenlider s. Lider

Augenmuskeln, erbliche Affek-tionen der - 37

Augenwimpern, Erkrankung der Augen nach Färbung der --318

B

Bacillus anthracoides 295 Bacillus subtilis 294 Bacillus tetragenus 295 Bacterium coli 294

Bakteriologie 291 (Bericht) Behrsches Syndrom bei Ver-

änderungen in der Fissura orbi-

talis superior 288 Beidäugiges Sehen 238 Beleuchtung, untertägige - in

Steinkohlenbergwerken und Nystagmus der Bergleute 239 Benzinvergiftung 314 Berlinsche

Trübung, Gefäß-

schatten bei - 233 Bindehaut s. Conjunctiva Bleivergiftung 315 Blepharitis, Behandlung der - 
mit Brillantgrün 114 Blindenwesen 228 Blutdruck der Netzhautgefäße

212 - und Augendruck 215 Blutgefäße, Nervenversorgung

der - 143 Blutgruppen bei Glaukom 312 Blutgruppentheorie, Bernstein-

sche 48 Blutkammerwasserschranke

209 Blutuntersuchungen am Auge

213 Botulismus 316 Brillantgrün zur Behandlung der

Blepharitis 114 Brillenkunde 52 (Bericht) Bulbuswand, Größenschwan-

kungen im Bereich einer trichter-

förmigen Ausbuchtung der hin-

teren -356

Sachregister zu Band 83.

371

Buphthalmus, pathologische Anatomie des $\mathbf{\square}-27$

C Calcarina, histologische Verände-rungen in der - bei Eklampsie

243 Carbaminoylcholin bei Glau-

kom 116, 117, 216 Cephalosporium 303 Chaulmoograöl bei Trachom 115 Chemie,

physiologische und physi-

kalische - 205 (Bericht) Chininvergiftung 315 Cholesterinkristalle in der Vor-

derkammer 236 Chorioidea, Biologie der intra-

okularen Sarkome der $\mathbf{-}-238$ Chorioretinitis mit Lochbildung

in der Macula 137 Conjunctiva, erbliche Verände-

rungen an der $\mathbf{a}-36$ - allergische Reaktionen der-

308 Conjunctivallappen, Anlegung

eines vorbereiteten - zur Ver-

hütung der Wundsprengung

nach Altersstarextraktion 87 Conjunctivitis, Koch-Weekssche

Bazillen - 251 Cornea, Durchmesser der -41

Pathologie der - 343 (Bericht)

Vererbung von Affektionen der

$-42$

- $\quad$-Uberpflanzung 120, 121

D

Dakryocystorhinostomie 125 Dämmerungszittern der Augen

138, 172 Denigsche Schleimhautpla-

stik 352 Diabetes mellitus 253

- Refraktionsänderungen bei -201

Diathermie 127

Gefahren der Nadel- 321

diath. Stichelung bei Netzhaut-abhebung 95, 189, 234

Diocain, augendrucksteigernde

Wirkung des - 331 Dioptrik 52 (Bericht) Diphtheriebazillen 294 Doryl, Drucksenkung durch 117, 216 Druck, intraokularer 214

experimentelle Steigerung des- 240

Steigerung des - durch Diocain 331

Drusenbildungen des Sehner-venkopfes 242 
i Dysostosis craniofacialis 48 Dystrophie, myotonische - und Katarakt 43

E Echinokokken 304 Eigenblutinjektionen in die

Vorderkammer bei Iristuber-

kulose 116 Einschlußkörper 299 Eisensplitter, 12 Jahre im Auge

ohne Verrostung 139 Eklampsie, histologische Verän-

derungen in der Calcarina bei -

243 Ektopia lentis, Vererbung ange-

borener - 37 Ektropium, Operation des Nar-

ben- 119 Elektrokoagulation 128 Elektrolyse (Kathoden-) zum

Auffinden und Verschluß des

Netzhautrisses 322 Elektronenröhre, Nachweis von

Metallsplittern im Körper mit

Hilfe der - 287 Embolie 319

Endotheldystrophien 179 Endothermie bei Ablatio retinae

124 Entropion, erbliches 36 -- Alkoholinjektionen in das Lid

bei - 114 Epiblepharon, erbliches 36 Episkleritis, Röntgenbestrah-

lung bei - 354 Epitheldystrophien 179 Erbliche Augenleiden, Verhü-

tung der - 248 Ernährung des Auges 209 Eutirsol 115 Extraktion, intrakapsuläre 122

$\Gamma$

Fädchenkeratitis 349 Fadenlampe, vereinfachte 35 Farbenasthenopie 132 Farbenblindheit,

Nystagmus bei

totaler - 1 Farbensinn, erbliche Störungen

des - 44 Farbenuntüchtiger Maler, 01-

bilder eines - 236 Fettembolie des Auges 252 Feuerarbeiter, Spaltlampen-

mikroskopie bei - 253 Fieberbehandlung bei tabischer

Sehnervenatrophie 117 Filaria 305 I Fissura orbitalis superior,

Behrsches Syndrom bei Ver-

änderungen in der $\mathbf{-}-288$

$25^{*}$

372

Sachregister

zu Band 83.

Flüssigkeitswechsel 209 Fremdkörperverletzungen 220 Frostschädigungen der Horn-haut 350

G

Gasbacillus 294 Gefäßschatten bei Berlinscher

Trübung 233 Gef ühllosigkeit, schmerzhafte -

bei Geschwülsten an der Spitze

der Orbita 73 Gehirntumoren, Hypophysen-

symptome bei -243

- $\quad$ Quecksilberbehandlung bei -366

Geschwülste, Schmerzsyndrom bei - an der Spitze der Orbita

73 -- Behandlung intrakranieller -

241 Germanin bei Pemphigus 74 Glaskörper, Antigen des - 309

- $\quad$ zystisches Gebilde im - 357Glaskörperblutungen, Rönt-

genbehandlung bei - 237 Glaskörperstoffwechsel 209 Glaukom, Vererbung des - 39 
erbliches jugendliches 317

familiar auftretendes -364

Blutgruppen bei -312

- $\quad$ nach Embolie der Netzhaut-arterien 138

- Carbaminoylcholin bei - 116Glaukosan, Iontophorese mit -

127 Glioma retinae bei 2 Brüdern 40 Gonokokken 293 Gonorrhoe, Komplementablen-

kungsreaktion bei - 311 Gunnsches Phänomen 37

$\mathrm{H}$

Hackensplitterverletzungen

221 Haftgläse |r 59, 60 Hefezellen der Bindehaut bei

Heufieber 303 Hemikinesimetrie 246 Herpes, ungewöhnlicher Verlauf

einer -infektion des Auges 67 Herpes corneae 297, 347

- $\quad$ traumatischer 226Heterochromie 38Heufieber, Hefezellen der Bindehaut bei - 303

Histamin, Drucksenkung nach -

216 Hornersches Symptom 38 Hornhaut s. Cornea Hydrophthalmus 343

Hydrophthalmus, Vererbung des angeborenen - 39

- $\quad$ und Krukenbergsche Pigment -spindel 70

Hypertonie, erbbiologische Ver-

hältnisse bei - 48 Hypophyse, Einwirkung der -•

auf den Augendruck 217

- $\quad$ Retinitis pigmentosa mit hypo-ph. Hochwuchs 354

Hypophysengeschwülste 368

- $\quad$ Operation der - 133Hypophysensymptome bei

Hirntumoren 243 Hypopyon-Uveitis, beiderseiti-ge rezidivierende - 357

I

Idiotie, familiäre amaurotische 45

- $\quad$ mongoloide, Augenveränderun-gen bei - 43

Immunität 306 (Bericht)

- $\quad$ und Anaphylaxie 246Infektionen, ektogene - des

Auges 291 Innere Sekretion und Myopie

199, 200 Insekten auf der Bindehaut 306 Iontophorese 127 Iridozyklitis, doppelseitige und Poliosis 71

- bazilläre Ätiologie mancher post-traumatischer Fälle von - 145

Iris, erbliche Affektionen der - 43 - Pigmentzysten der Hinterfläche der - 135

- $\quad$ Eigenblutinjektionen in die Vor-derkammer bei Tuberkulose der- 116

Iristuberkulose, hochgradige allergische Phasen im Verlaufe der - 257

K

Kammerwasser, Analysen des -

206 Katarakt, erbliche $43 \mathrm{Ka}$ tar a kt operation, Sicherungs-

maßnahmen bei - 249

eines Röntgenstars 260

Wundsprengung nach Alters-starextraktion und ihre Ver-hütung 87

Kathoden-Elektrolyse zum Auffinden und zum Verschluß des Netzhautrisses 322 Keratitis mit

Psoriasis 66 Keratitis parenchymatosa 345

- $\quad$ und Trauma 219

Scharlachsalbe bei - 116 Keratokonus 344 
bei Geschwistern 42

Sachregister

zu Band 83.

373

Keratoplastik 351

Kn ochenbrüchigkeit und blaue

Sklera 43 Koch-Weekssche Bazillen25i,

294 Kohlengasvergiftung 316 Kolobome, Vererbung von - 37 Kopfschmerzen und schmerz-

hafte Gefühllosigkeit bei Ge-

schwülsten an der Spitze der

Orbita 73 Kraftfahrer, Schutzbrillen für-

239

L

Lash-luve, Augenerkrankung nach Wimperfärbung mit - 318

Laurence-Biedlsches Syn-drom 46

Lebersche Krankheit 365

Lenticonus posterior 355

Leptothrix 303

Linse, Form der $\mathbf{\square - 5 3}$

Dicke der - $\square$ und Refraktion 201

Kalkgehalt der - 208

-- erbliche Veränderungen der -

36, 43 Lysozym 313

$\mathrm{M}$

Macula, Degeneration der -46

- $\quad$ Lochbildung in der - bei Cho-rioretinitis 137

Malen, wie malten sich die alten Meister selbst ? 74 (Bb.)

Maler, Ölbilder eines farbenun-tüchtigen - 236

Marfanscher Symptomenkomplex

37 Maul- und Klauenseuche mit

Beteiligung des Auges 297 Medikamentöse Therapie 114

(Bericht) Megalocornea 42, 343 Meningiome, parasagittale 368 Menformon, Erblindung durch 316 Metallsplitter im Körper, Nach-

weis der - mit Hilfe der Elek-

tronenröhre 287 Methylbromid, Vergiftung mit

$-314$

Migraine ophthalmique, Ace-

tylcholin bei - 118 Mineralbestand des Auges 205 Molluskum contagiosum 297 Musculus

obliquus inferior,

Myektomie des - 134 Myopie 41, 198

- $\quad$ Beziehung der Fundusdegene-ration bei Anisometropie zurGenese der a- 252

N Nadeldiathermie, Gefahren der

$-\quad 321$

Naevus flammeus und Glaukom

40 Narbenektropion, Operation des 
-- 119 Natriumsalizylat, Wirkung des

intravenös gegebenen - 16 Nervenversorgung der Blutge-

fäße 143 Nervus oculomotorius, perio-

dische Lähmung des - und

Tuberkulose 7 Nervus opticus, erbliche Affek-

tionen des - 48 Nervus opticus-atrophie, he-

reditäre 365 - auf angiospastischer Grundlage

$7 \mathrm{i} \bullet-$ retrobulbäre Atropineinsprit-

zungen bei - 117, $134 \cdot-$ Fieberbehandlung der tabischen

- $\quad 117,118$ Netzhaut s. RetinaNetzhautablösung s. Ablatio

retinae Neuritis retrobulbaris durch

Fokalinfektion von den Zähnen

aus 244 Nipasol 114 Nystagmus bei totaler Farben-

blindheit 1

optokinetischer - bei Septo-jodschädigung der Netzhaut 338

der Bergleute und untertägige Beleuchtung in Steinkohlenberg-werken 239

0

Oguchische Krankheit 45 Operationszwang bei Unfallbe-

schädigten 226 Operative Therapie 119 (Ber.) Ophthalmia sympathica, ba-

zilläre Ätiologie der - 145, 168

- $\quad$ Tuberkelbazillenkultur aus demGewebe des symp. Auges 163,296

- Beurteilung des histologischen Befundes im zweiten symp. er-krankten Auge 77

- Atophanyl bei - 117Optik, physikalische 52 (Bericht)Oslersche Krankheit in 3 Gene-

rationen 48

$\mathrm{P}$

Pannus, Operation des - 119 Pantocain zur Anästhesierung bei der Tonometrie 331

374

Sachregister

zu Band 83.

Pantocain, Ekzem der Lider

nach Betäubung mit - 112 Parasiten, tierische 304 Pemphigus, Germanin bei - 74 Pemphigus

conjunctivae 139 Phakomatosen 47 Phlyktäne 310 Physikalische Therapie 127

(Bericht) Pigmentierung, Vererbung von

Anomalien der - 38 Pigmentspindel, Krukenberg-

sche - und Hydrophthalmus 70 Pigmentzysten der Irishinter-

fläche 135 Pikrotoxinvergiftung 314 Pilze der Bindehaut 302 Pneumokokken, augenpatho-

gene Bedeutung der - 250, 293 - Immunisierung gegen - 312 Poikilodermatomyositis, Au-

generkrankungen bei - 254 Poliosis und doppelseitige Irido-

zyklitis 71 Presbyopie 203 Prowazek-Halberstädtersche

Epitheleinschlüsse 299 Pseudosklerose Wilson 48 Pseudotumoren des Gehirns 136 Psoriasis mit

Keratitis 66 Pterygiumoperation, rezidiv-

verhütende 100 Ptosis, erbliche 37 Pupillenbewegungen 255 (Bb.) Pupillenstarre, scheinbare -

mit Fehlen von Sehnen- und

Periostreflexen 278 Pupillotonie 278

Quecksilberbehandlung bei Hirntumoren 366 
$\mathrm{R}$

Radiumbehandlung 128 Reflexbilder des Auges, Stereo-

photogrammetrie der - 245 Refraktion 40

- Anomalien der - 198 (Bericht) Refraktionsbestimmung 52

(Bericht) Retina, erbliche Anomalien der -

, 44

Retinaarterien, Blutdruck der

-212 - Embolie der - mit anschließen-

dem Glaukom 138 Retinariß, Auffinden und Ver-

schluß des - durch Elektrolyse

322 Retinitis, schwere einseitige -

355

Retinitis pigmentosa, erbliche

42, 46 - mit multiplen Mißbildungen und

hypophysärem Hochwuchs 354 Rickettsia orientalis 297 Ringgebilde, eigenartige -in der

Vorderkammer 70 Röntgenbehandlung 128 Röntgenstar, Extraktion eines -

mit Zysteinreaktion in der Linse

260

S Sarkome, Biologie der intraoku-

laren - der Chorioidea 238 Scharlachsalbe bei Keratitis

parenchymatosa 116 Schielen als traumatische Neurose

137

- $\quad$ Fadenfixation des Bulbus bei -

239

- $\quad$ stereoskopische Bilder für Schie-lende 256 (Bb.)

Schmerzsyndrom bei Geschwül-sten an der Spitze der Orbita 73 Schutzbrillen für Kraftfahrer

239 Schwefelkohlenvergiftung3i4 Sehnerv s. Nervus opticus Sehproben 62 Sehschwache Kinder

229 Septojodschädigung der Netz-haut, optokinetischer Nystagmus bei - 338 Serologie 306

(Bericht) Sklera, Bau der - 353

- $\quad$ blaue - und Knochenbrüchig-keit 43

- $\quad$ Ruptur der - 353Skleritis, pathologische Anatomie

der - 324 Sklerose, multiple; Erbanlage

bei - 48 Skrofulöse Augenerkrankung

3 i8 Soziale Medizin 223 Spaltlampenmikroskopie bei

Feuerarbeitern 253 Spirochäten 294 Staphylokokken im Auge 292 Status dysraphicus 38

Stereophotogrammetrie der

Reflexbilder des Auges 245 Sterilisierung von Augentropfen

114 Streptokokken, augenpathogene

Bedeutung der - 250 Sulfosinzur Fieberbehandlung der

Sehnervenatrophie 118 Synechien, vordere - und Zyklo-

dialyse 238 Syphilis, Intradermoreaktion bei

-3 "

Sachregister zu Band 83.

375 
Tätowage 351

- $\quad$ von Hornhautnarben 121Tetrakohlenst off ver gift ung

314 Thalliumvergiftung 315 Therapie, ophthalmologische 114

(Bericht) Thorium X bei Xanthelasma 129 Thrombose 319 Tonometrie, Pantocain zur An-

ästhesierung bei der - 331 Totische Operation 125 Trachom 298, 299, 312, 350

- $\quad$ Verbreitung des - in Deutsch-land 263

- $\quad$ Behandlung des - 115, 119Tränendrüsen und Allgemein-

erkrankungen 247 Trypanosomen 305 Tuberkelbazillen im Auge 296 a- Kultur von - aus dem

Gewebe

des sympathisierenden Auges

163 Tuberkulose, Immunität und Al-

lergie bei $\square-\square 309$

- $\quad$ und periodische Oculomotorius-lähmung 7

-- tub. exsudative Entzündung des Augeninnern 362

U Ulcus rodens 349

- $\quad$ Bindehautdeckung bei - 122Ulcus serpens 349

- in landwirtschaftlichen Betrie-

ben 221 Uricedin bei gichtischen Augen-

erkrankungen 117 Uvea, Allergie der - 309

V Vakzinebehandlung des Tra-

choms 115 Vegetative Physiologie und

Pathologie 205 (Bericht)

Verätzungen 219 Vererbung und Auge 36 (Bericht) Vergiftungen 314 Verletzungen 219

(Bericht) Versicherungswesen 223 Vierhügel, Tumor der -· 138 Visolet-Lupe 69

Vorderkammer, Tiefe der - 41 - eigenartige Ringgebilde in der - 70

$\mathrm{W}$

Wärmebehandlung bei Horn-

hauterkrankungen 128 Wimperfärbung, Augenerkran-

kung nach - 318 Wismutvergiftung 315 Wundsprengung nach Alters-

starextraktion und ihre Ver-

hütung 87

$\mathrm{X}$

Xanthelasma, Thorium X-Be-

handlung bei - 129 Xeroderma pigmentosum bei Ge-

schwistern 40

Y

Yatoconineinspritzungen bei Trachom 115, 350

Z

Zähne, Neuritis retrobulbaris

durch Fokalinfektion von den -

aus 244 Zeissspatlampengerät nach

Comberg 239 Zeozonwasser 115 Zilien, Form und Farbe der - 36 Zwillingsuntersuchungen 48

Zyklodialyse und vordere Sy-

nechien 238 - Netzhautablösung als Folgezu-

s. stand nach - 233 Zysteinreaktion in der Röntgen-

starlinse 260 Zystizercus 304. 
